\title{
A Novel Method to Detect the Frame-Formats in 802.11n
}

\author{
Yong Zhu \\ Jiangsu Automation Research Institute, Lianyungang, 222061, China \\ email: yaoxiaocheng@jari.cn
}

Keywords: OFDM, frame format differentiate, TGn models, MIMO

\begin{abstract}
There are three different frame formats in standard IEEE 802.11n which are mixed format HT (high throughput), non-HT and green field HT. Two types of signal were proposed in different frame formats, HT-SIG and L-SIG. Since both L-SIG and HT-SIG are BPSK modulated, the HT-SIG is rotated by 90 degrees relative to L-SIG. The rotation allows for robust auto-detection between L-SIG and HT-SIG to differentiate varied frame formats. We proposed a novel method which employs the channel information to weigh the subcarrier detected results with high accuracy and low complexity compared to the conventional methods. The mathematical analysis and simulation results demonstrate that the proposed algorithm can achieved the nearly maximum-likelihood detection performance which is more than $10 \mathrm{~dB}$ better than the method suggested in [4] at the differentiation error ratio of $10^{-2}$.
\end{abstract}

\section{Introduction}

Wireless local area networking has experienced tremendous growth in the last ten years with the proliferation of IEEE 802.11 device. A milestone event for commercial Wireless Local Area Networks (WLANs) came about in 1985 when the United State Federal Communications Commission (FCC) allowed the use of the experimental Industrial, Scientific, and Medical (ISM) radio bands for the commercial application of the spread spectrum technology. Several generations of proprietary WLAN devices were developed to use these bands. The initial systems were expensive and deployment was only feasible when running cable was difficult.

Advances in semiconductor technology and WLAN standardization with 802.11 led to a dramatic reduction in cost and the increased adoption of WLAN technology. WLANs offer many benefits over wired networking. In existing homes or enterprises, deploying cables for network access may involve tearing up walls, floors, or ceilings, which is both environment and costly. In contrast, providing wireless network connectivity in these environments is often as simple as installing a single wireless access point. Perhaps more importantly though, the proliferation of laptops and handheld devices has meant that people desire connectivity wherever they are located, not just where the network connections are located. Network connectivity in a conference room or while seated on the sofa in the living room are just two examples of the flexibility afforded by WLANs [1 3].

The vast majority of WLAN products and systems today are based on the $802.11 \mathrm{~b}, 802.11 \mathrm{~g}$, and 802.11a standard and up to today 802.11n standard which provide throughput enhancements over the original 802.11 PHYsical layers (PHY). Market drivers were outlined, such as increasing data rates of wired Ethernet, more data rate intensive applications and the need for higher capacity WLAN networks. The presentation mentioned techniques such as spatial multiplexing and doubling the bandwidth as potential approaches which are exploited in IEEE 802.11n to study for increasing data rate. The data rate can reach up to 600Mbps in the present IEEE 802.11n standard.

Since IEEE 802.11n should permits the legacy compatibility and achieving high rate throughput, there are three different frame formats [4]: Mixed Field HT (MF-HT), Green Field HT (GF-HT), NON-HT as illustrated in figure 1.

-NON-HT: Packets of this format which are structured according to IEEE 802.11a/g (OFDM) is mandatory in IEEE 802.11n.

- HT-MF: Packets of this format contain a preamble compatible with packets of Non-HT format. As shown in figure 1, the non-HT-STF (L-STF), the non-HT-LTF (L-LTF), and the non-HT 
SIGNAL field (L-SIG) are defined so they can be decoded by non-HT receivers. The rest of the packet cannot be decoded by non-HT receivers. Support for HT-mixed format is mandatory in 802.11n.

-HT-GF: HT packets of this format do not contain a non-HT compatible part. Support for HT-GF is optional in IEEE 802.11n. A HT receiver that does not support the reception of an HT-GF packet shall be able to detect that an HT-GF packet is an HT transmission (as opposed to a non-HT transmission). In this case, the receiver shall decode the HT-SIG and determine whether the HT-SIG Cyclic Redundancy Checked (CRC) passes.

As illustrated in Figure 1, the HT portion of the MF preamble consists of High Throughput Signal (HT-SIG) field, including HT-SIG1 and HT-SIG2, which contain new signaling information and are also used for auto-detection between different frame formats. It usually contains two steps to complete differentiating different frame format in IEEE 802.11n. First step, during16us-20us as illustrated in Figure 1, is to detect whether the coming signal is L-SIG or HT-SIG to differentiate HT-GF and other formats. If the coming signal is L-SIG, then the coming frame may be HT-MF or NON-HT, otherwise is HT-GF. Second step, if the coming frame is HT-MF or NON-HT and the data rate indication is 6Mbps as defined in IEEE 802.11a/g known after the first step, during 20us-24us as illustrated in Figure 1, is to detect whether the coming data is legacy data or HT-SIG2. If the coming data is legacy data, then the coming frame is NON-HT, otherwise is HT-MF.

\begin{tabular}{|c|c|c|c|}
\hline$\overbrace{}^{8 u s}$ & $\overbrace{}^{8 \mathrm{us}}$ & $\overbrace{}^{4 u s}$ & \\
\hline L-STF & L-LTF & $\begin{array}{c}\text { L- } \\
\text { SIG }\end{array}$ & Data \\
\hline
\end{tabular}

\begin{tabular}{|c|c|c|c|c|c|c|c|c|c|c|c|}
\hline \multicolumn{5}{|c|}{ HT-mixed format PPDU } & \multicolumn{3}{|c|}{$\begin{array}{l}\text { Data HT-LTFs } \\
\text { 4us per LTF }\end{array}$} & \multicolumn{3}{|c|}{$\begin{array}{l}\text { Extension HT- } \\
\text { LTFs 4us per LTF }\end{array}$} & \\
\hline L-STF & L-LTF & \begin{tabular}{|l} 
L- \\
SIG
\end{tabular} & HT-SIG & $\begin{array}{l}\text { HT- } \\
\text { STF }\end{array}$ & $\begin{array}{l}\text { HT- } \\
\text { LTF }\end{array}$ & & \begin{tabular}{|l|} 
HT- \\
LTF
\end{tabular} & $\begin{array}{l}\text { HT- } \\
\text { LTF }\end{array}$ & & \begin{tabular}{|l|} 
HT- \\
LTF
\end{tabular} & Data \\
\hline
\end{tabular}

\begin{tabular}{|c|c|c|c|c|c|c|c|c|}
\hline \multicolumn{3}{|c|}{ HT-greenfield format PPDU } & \multirow{2}{*}{\multicolumn{3}{|c|}{$\begin{array}{l}\text { Data HT-LTFs } \\
\text { 4us per LTF }\end{array}$}} & \multirow{2}{*}{\multicolumn{2}{|c|}{$\begin{array}{l}\text { Extension HT- } \\
\text { LTFs 4us per LTF }\end{array}$}} & \\
\hline 8us & 8us & 8us & & & & & & \\
\hline L-STF & L-LTF & HT-SIG & $\begin{array}{l}\text { HT- } \\
\text { LTF }\end{array}$ & $\cdots$ & $\begin{array}{l}\text { HT- } \\
\text { LTF }\end{array}$ & \begin{tabular}{|l|} 
HT- \\
LTF
\end{tabular} & \begin{tabular}{|l|l|}
$\cdots$ & HT- \\
LTF \\
\end{tabular} & Data \\
\hline
\end{tabular}

Fig. 1. PPDU Format in the IEEE 802.11n

In section II we present a brief overview of original differentiation methods. A novel method which employs the channel state information is introduced in Section III. Detailed simulation results of the proposed method compared with the conventional method over frequency selective channels are displayed in Section IV. Finally we end the paper with a brief conclusion in Section V where we restate the important themes presented.

\section{Overview of Conventional Algorithm}

Since both the L-SIG and HT-SIG are Binary Phase Shift Keying (BPSK) modulated, the HT-SIG is rotated by 90 degrees relative to L-SIG, as illustrated in Figure 2.
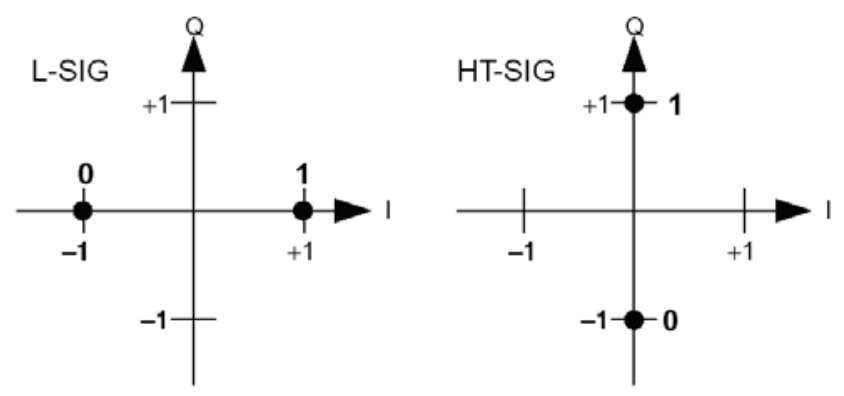

Fig. 2. Data Subcarrier Constellation of L-SIG and HT-SIG

The rotation allows for robust auto-detection between L-SIG and HT-SIG1 to differentiate between legacy OFDM packets and HT-MF packets. 
An example auto-detection algorithm is as fellows. For each symbol that is processed, compute a metric based on the power of the real part of the data subcarriers averaged over the 48 data subcarrier minus the power of the imaginary part of the data subcarriers averaged over the 48 data subcarriers as described in Eq.(1). Where the $D_{\text {original }}$ is the detection result, $\hat{x}(i)$ is the $i$ th data subcarrier after frequency domain equalization. With an appropriate threshold, we can differentiate between a legacy OFDM packet and a HT mode packet.

$$
D_{\text {original }}=\sum_{i=1}^{N_{D}}|\operatorname{Re}(\hat{x}(i))|^{2} / N_{D}-\sum_{i=1}^{N_{D}}|\operatorname{Im}(\hat{x}(i))|^{2} / N_{D}
$$

Employing the thought of Maximum likelihood, the threshold is set to 0 . That means that if $D_{\text {original }}<0$, the received symbol is HT-SIG, otherwise it is not. There are some other similar

algorithms such as employing amplitude instead of the power as shown in Eq.(2), where $D_{\text {modified }}$ is the detected result of the modified method.

$$
D_{\text {modified }}=\sum_{i=1}^{N_{D}}|\operatorname{Re}(\hat{x}(i))|-\sum_{i=1}^{N_{D}}|\operatorname{Im}(\hat{x}(i))|
$$

The algorithms referred above suppose each data subcarrier with the same fading factor. That is equal to say that it may have good performance when the receiver with the propagation modeled by flat fading channel or on high signal to noise ratio (SNR).In practical implementation, the WLAN propagations are always modeled in fast fading channel or frequency selective channel. In order to improve the performance of the frame differentiation in the low SNR and frequency selective channel, a novel algorithm needs to be proposed.

\section{Proposed Algorithm}

The system of IEEE 802.11n with single transmit antenna and single receive antenna can be basically described as Eq.(3).

$$
y=h x+z
$$

where $x$ is the transmitted data with a fixed mean expected power, $h$ is the channel fading coefficient, $Z$ is independent, complex additive white Gaussian noise (AWGN) with zero mean and fixed variance for a special system, and $y$ is the received signal. Typically $h$ is modeled as Rayleigh fading channel coefficient. In the frequency domain, each subcarrier may be described by Eq.(3). Subsequently, the receiver extracts the information by equalizing the received signal described as Eq.(4).

$$
\hat{x}=y / \hat{h}=(h x+z) / \hat{h}
$$

where $\hat{x}$ is the estimate of the transmitted data $x, \hat{h}$ is the estimate of the channel coefficient $h$.

For the HT-SIG is rotated 90 degree compared to L-SIG, it is advisable to detect each subcarrier and then combine all the subcarriers detect result to differentiate whether the processing symbol is HT-SIG or not. First, we can compare the power of the real part and the image part of each subcarrier. A simplified method with same performance that employs amplitude instead of power is proposed described as Eq.(5).

$$
\text { Sub_Detect }(i)=(|\operatorname{Re}(\hat{x}(i))|>|\operatorname{Im}(\hat{x}(i))|) * 2-1
$$

where $\operatorname{Re}(\hat{x}(i))$ is the real part of the $i$ th subcarrier data and $\operatorname{Im}(\hat{x}(i))$ is the image part of the $i$ th subcarrier, Sub_Detect(i) is the $i_{\text {th }}$ data subcarrier detected result. We can conclude from Eq.(5) that $S u b_{-} \operatorname{Detect}(i)$ is 1 when $|\operatorname{Re}(\hat{x}(i))|>|\operatorname{Im}(\hat{x}(i))|$, otherwise Sub_Detect(i) is -1 . The Eq.(5) can simplified the complexity of the implementation for the following stages.

Second step, compute the sum of the each subcarrier detection result to eliminate the noise effect. 
The detect result can be got as follows:

$$
D_{\text {simple }}=\sum_{i=1}^{N_{D}} S u b_{-} \operatorname{Detect}(i)
$$

where $D_{\text {simple }}$ is the differentiate result of the simple method. The received symbol is considered to be HT-SIG if $D_{\text {simple }}<0$, else it is L-SIG or L-DATA.

In an IEEE 802.11n system the channel estimation $\hat{h}(i)$ is already calculated using the long training sequences in the preamble for equalization. In some case, the magnitude of the channel estimation $|\hat{h}(i)|$ is already available. In order to reduce the noise affection, the optimal method is proposed as Eq.(7).

$$
D_{\text {optimal }}=\sum_{i=1}^{N_{D}}|\hat{h}(i)| * S u b_{-} \operatorname{Detect}(i)
$$

where $D_{\text {optimal }}$ is the differentiate result of the optimal method, if the $D_{\text {optimal }}<0$, it demonstrates that the received symbol is HT-SIG, otherwise, it is not.

Note that for channels without frequency selective fading, the expect value of the $\hat{h}$ is the same for all subcarriers in OFDM and therefore there is no difference in the performance of the Eq.(6) and Eq.(7). When there is frequency selectivity in the channel, however, different subcarriers are weighed differently. Since weighing provides the modification to the subcarriers detect result, optimum differentiation performance is achieved.

For the receiver of the IEEE 802.11n system with multiplex antennas, the method above needs to be improved. The above method can be considered as one antenna data flow processing. The final detection result can be acquired by combining all the antennas data flow detect result described as Eq.(8).

$$
D_{\text {mimo }}=\sum_{j=1}^{N_{r x}} \sum_{i=1}^{N_{D}}|\hat{h}(j, i)|^{*} \operatorname{Sub} \_\operatorname{Detect}(j, i)
$$

where the $D_{\text {mimo }}$ is the final detect result, $\hat{h}(j, i)$ is the $i$ th subcarrier $j$ th antenna channel estimation coefficient, $\operatorname{Sub} b_{-} \operatorname{Detect}(j, i)$ is the $i$ th subcarrier $j$ th antenna detect result, $N_{r x}$ is the number of the receiver antennas. The Eq.(8) can also be described as E.q.(9).

$$
D_{\text {mimo }}=\sum_{j=1}^{N_{\text {rx }}} D_{\text {optimal }, j}
$$

where $D_{\text {optimal, } j}$ is the $j$ th antenna data flow detect result which can be computed as the SISO referred above.

Since the proposed method employs the channel message to weigh the subcarrier detect result and eliminates the multiplication, the proposed method not only has high performance but also easily to implementation with less source consumption.

\section{Simulation Results}

The functional simulation is under TGn Channel Models [6][7] which are varied typical channel model. The center frequency is set to $5320 \mathrm{MHz}$. The Carrier Frequency Offset (CFO) and Sampling Clock Offset (SCO) are both -13.65ppm according to the Task Group 802.11n (TGn) comparison criteria. The reference channel models in simulation are described in table 1. 
Table 1. TGn Channel Model Description

\begin{tabular}{|l|l|l|l|}
\hline Model & RMS (ns) & Environment & Example \\
\hline B & 15 & Residential & Intra-room, room to room \\
\hline D & 50 & Typical Office & Intra-room, room to room \\
\hline E & 100 & Large Office & Large Conference, Hotel \\
\hline
\end{tabular}

We ran simulation on IEEE 802.11n in HT-MF and NON-HT mode for 10000 packets on different simulation condition. We compared the performance of the conventional method and two method proposed in the paper.

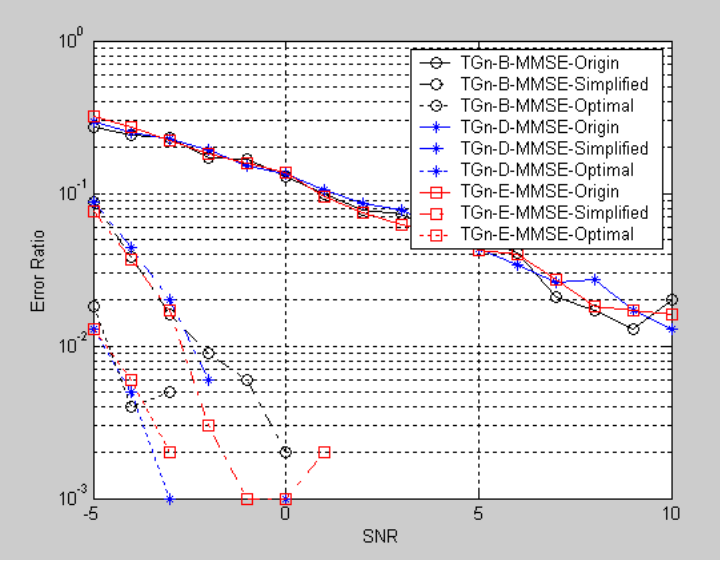

(a) The Performances on MMSE Channel Estimation

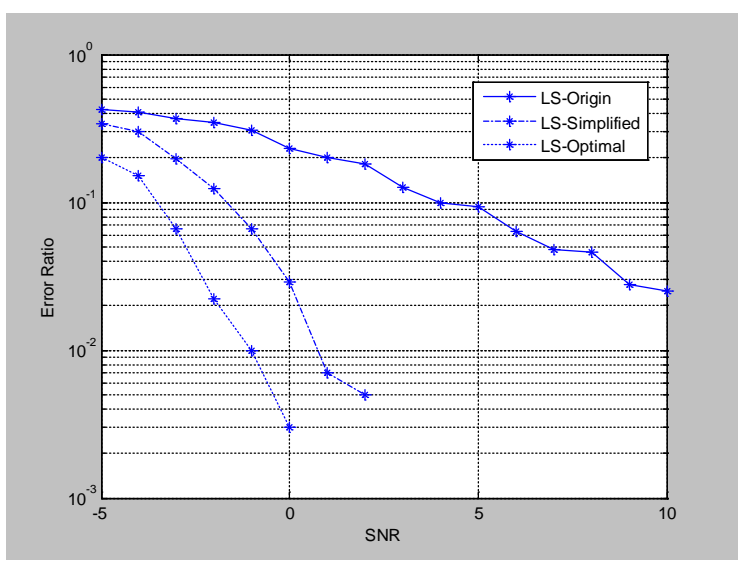

(b) The Performances on LS Channel Estimation

Fig. 3. The Performances on Varied Channel Estimation and Channels

For TGn channels (B, D, E) and the channel estimation employing Minimum Mean Square Error (MMSE) method [8,9] the performances of the three different methods are shown in Figure 3(a). As can be seen from Figure 3, the performance of the proposed method is slightly better than the origin method. For TGn channels the improvement of the channel SNR is more than $10 \mathrm{~dB}$ for both simplified method and optimal method at the differentiation error ratio of 10-2 and more than $10 \mathrm{~dB}$ at differentiation error ratio of $10^{-3}$. It can also be seen from the Figure 3 that the improvement of the channel SNR for optimal method is about 2 3 dB compared to the simplified method. Over different TGn channel type, the performance has little difference. It can be concluded from the simulation result that the performance has little to do with TGn channel type.

The performances that channel estimation employing LS method [10] are shown in Figure 3(b). It can be seen that the improvement of the channel SNR is similar to that shown in Figure 3(a). The simulation result shown in Figure 4 demonstrated that the performances of simplified and optimal method are good even in low SNR and for system employed simple channel estimation. In that case, the method proposed will not be a roadblock of the system design.

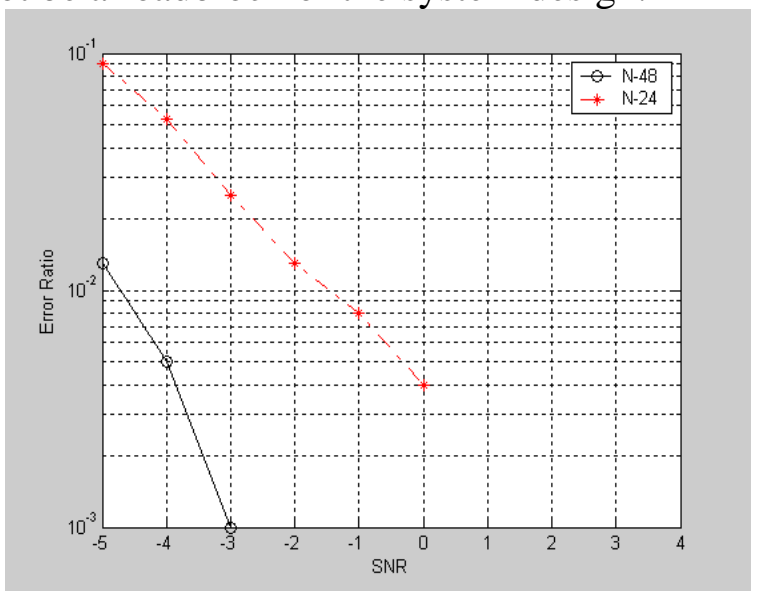

Fig. 4. The Performances of Varied Length of Data Signals VS SNR

The complexity can be reduced by reducing the length of the computed signal data. The performance of the optimal method that employs only half of the length of the signal data compared to that employ the total data was shown in Figure 5. It can be seen that the loss of channel SNR is 
about $3 \sim 4 \mathrm{~dB}$ compared to that employs the total signal data. So it supplies more smart process for system design.

\section{Conclusion}

In this paper, we proposed a novel method to detect the frame format in 802.11n. The proposed method corrects the effect of frequency selective channel. Simulation results illustrated that the proposed method improved the performance of the method suggested in [2]. Further analysis showed that even with the accuracy channel estimate, the proposed method leads to a substantial improvement in performance of the receiver.

In a typical IEEE 802.11n receiver the channel information is already obtained using the long training field in the preamble for equalization. Therefore, the implementation of the proposed method not only introduced no significant computational complexity to the system, but also reduced the computation complexity for employing comparators instead of average power computation.

\section{Acknowledgement}

The work is supported by Natural Science Foundation (Project No. 61303045).

\section{References}

[1] IEEE Std 802.11nTM-2009: Available:http://standards.ieee.org/getieee802/download/802.11n -2009.pdf.

[2]Lin Shijun, Shi Jianghong. Carrier sensing rang analysis in general IEEE 802.11 network with physical-layer network coding, Wireless Communication and Mobile computing, v15 p2206-2218, 2015

[3] Thorpe Christina, Murphy Liam. A survey of adaptive carrier sensing mechanisms for IEEE 802.11 wireless networks, IEEE Communications survey and tutorials, v16, p1266-1293,2014

[4] Eldad Perahia and Robert Stacey. Next Generation Wireless LANs. Cambridge University Press, 2008.

[5] Enis Akay and Ender Ayanoglu. High Performance Viterbi Decoder for OFDM Systems.Vehicular Technology Conference, May17-19,Milan,Italy,2004.

[6] Erceg, V., Schumacher,l., Kyritsi, P., et al. TGn Channel Models, IEEE 802.11-03/940r4. Available:http://www.ieee802org/11/DocFiles/03/11-0300940-04-000n-tgn-channel-models.doc

[7] Thomas Paul and Tokunbo Ogunfunmi. Wireless LAN Comes of Age: Understanding the IEEE 802.11n Amendment, IEEE Circuits and System Magazine.

[8] Jingxian Wu, Chengshan Xiao. Optimal Diversity Combining Based on Linear Estimation of Rician Fading Channels, IEEE Communications Society. 2007.

[9] Manu. K.M.,Nelson.K.J..OFDM channel estimation using variation minimization in compressed sensing, International Conference on Contemporary Computing and informatics. 2014 1231 1234.

[10] Qing Haobo, Liu Yuan-an, et al. Parametric channel modeling based OFDM channel estimation. [J]. Journal of China Universities of Posts and Telecommunications, 2014 (21) 1-8 